\title{
Hunting for Ghosts: Low Surface Brightness Galaxies from Pixels
}

\author{
Roberto Scaramella ${ }^{1} \dagger$, and Sabina Sabatini ${ }^{1}$ \\ ${ }^{1}$ I.N.A.F. - Osservatorio Astronomico di Roma
}

\begin{abstract}
We outline an approach devoted to the detection of low surface brightness galaxies on astronomical images. We use a multi-scale approach, take into account sources of incompleteness and check selection functions both analytically and with simulations.
\end{abstract}

Keywords. Low Surface Brightness galaxies

\section{Introduction}

The capital importance of surface brightness selection effects in galaxy detection was brought to attention for the first time by Disney (1976) who argued that the knowledge of galaxies had been heavily biased by the sky background brightness - the detection of low surface brightness galaxies (LSBGs) being strongly penilised by these effects. The number of specific studies searching for this kind of objects increased significantly since then and the interest on the subject is still strong (for recent work see Kniazev et al. (2004) and Haberzettl et al. (2007)). Different surveys use different methods for the detection of LSBGs and are therefore subject to different selection effects, completeness function and contamination. This often makes the comparison of results in the literature quite difficult. Here we present an on going work to improve a dedicated package aimed at the detection of LSBGs on digital images, with a reasonably good control of systematics and incompleteness of the obtained galaxy catalogues. In the long term we plan to apply this method to surveys of different depths and environments in a consistent and homogeneous way.

\section{Method}

\subsection{Overview}

The detection algorithm has been applied to both digitized POSS plates and CCD mosaics and its structure can be summarized as follows: every image is first background subtracted and then cleaned from standard objects, that are detected by means of SExtractor (Bertin et al. (1996), hereafter SE); we then use a convolution technique to enhance the signal of extremely low surface brightness objects in the masked image. The convolution is performed with a stack of digital kernels of different scale lengths. This produces a list of detected candidates for each scale, which is then pruned for multiple detections according to significance, yielding an estimate of the scale length and a significance index for each candidate. The luminosity profiles of the candidates matching our search criteria are then fit to an exponential profile to have direct estimates of structural parameters like scale length, $\alpha$, and central surface brightness, $\mu_{0}$. The various steps of the process are described in the following sub-sections.

$\dagger$ Present address: Osservatorio Astronomico di Roma, via di Frascati 33, 00040 Monteporzio Catone (RM), Italy 
We remark that the algorithm presented here is tailored to detect only objects that are possible LSBG candidates. It is well known that one of the major difficulties in this is given by the fact that often a LSBG is fragmented by standard detection algorithms that use the approach of searching for connected pixels above a threshold. We therefore widely use such methods (SE in particular) only for the cleaning process (flat-field, background and bright sources subtraction), since they are highly reliable, fast and flexible in producing catalogs from easily customizable parameters. We check that all the faint objects detected by SE with typical parameters are also detected by our algorithm. The converse is not always true and it is mainly due to the use of convolution over a stack of kernels rather than with a single one as it is done in standard SE.

\subsection{Image cleaning and flat-fielding}

For handling purposes, each original image is divided in footprints of size $2048 \times 2048$ pixel, with a small overlap between adjacent footprints (usually 100 pixels), in order to properly handle the objects falling at the border of each footprint. An image (i.e. a footprint of a CCD mosaics or a plate scan) needs two preliminary steps before object detection. First of all, since we are looking for extremely faint objects, close to the sky noise, we need a good estimate of the sky background. Second, we need an objective criterion to select and remove all the bright objects present on the image.

1) We adopted a two stage pass for a robust background estimate. In order to get the background map and a catalogue of bright objects we use the package SExtractor. To prevent overestimating the background, in presence of highly saturated objects, bright stars (with possible long crosses and/or large haloes) or moderately crowded fields, a first pass of SE is run on the raw image and all the detected objects are masked in the image with random noise patches of level $\sigma_{s k y}$. On this masked image we run SE again and obtain a background map that is then used to "flat-field" the raw image. This procedure yields a sky-subtracted image that is ready to be analysed.

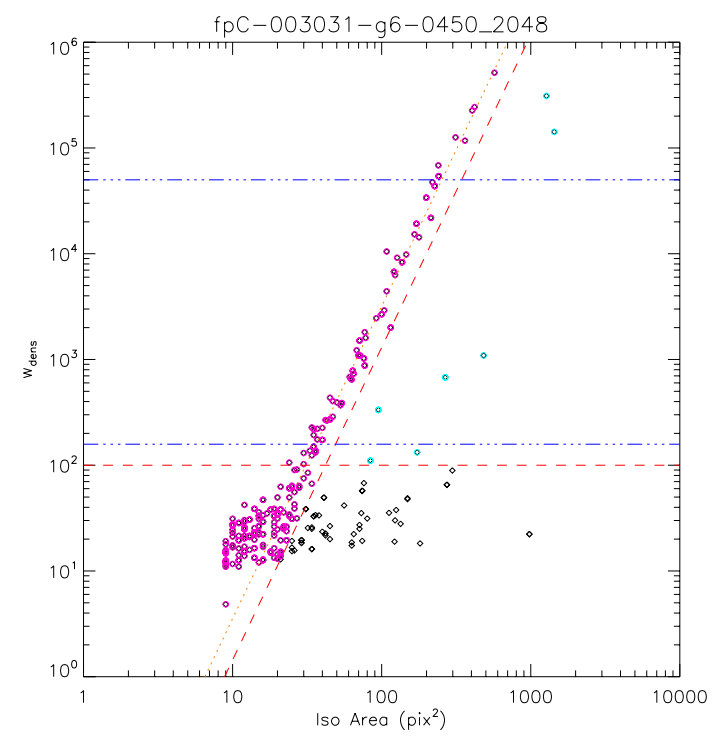

Figure 1: Object distribution on the $\left(W_{\text {dens }} A_{\text {iso }}\right)$ plane for a single SDSS footprint. The stellar locus is on the diagonal line. Large bright galaxies and faint ones and/or possible tips of LSB candidates occupy the region to the right of the stellar locus. 
2) After background subtraction we also need to discard objects that are not interesting for our investigation, i.e. stars, bright galaxies, plate/CCD defects, etc. For this purpose we use the SE output catalogue and select and mask those objects in the following way. We a plane defined by isophotal areas and magnitudes, where it is relatively easy to discriminate among objects of different nature and trace the stellar locus. In particular we use a $\left(W_{\text {dens }}, A_{\text {iso }}\right)$ plane (see Fig. 1$)$, where $W_{\text {dens }}$ is defined as $W_{\text {dens }}=f_{\text {max }}\langle f\rangle / \sigma_{\text {sky }}^{2}$ and $\sigma_{s k y}$ is the pixel sky variance, $f_{\max }$ is the peak flux above the background, $\langle f\rangle=$ $f_{i s o} / A_{i s o}$ is the average flux, $A_{i s o}$ is the isophotal area above the analysis threshold and $f_{i s o}$ is the isophotal flux. Each of these parameters are given by SE in its output catalogue.

In this plane, the object distribution depends on dimensions and flux shape observed above the SE analysis threshold. We can distinguish objects with large $W_{\text {dens }}$ at the top of the plot as saturated stars $\left(W_{\text {dens }} \approx 10^{5}\right)$ or bright and widespread objects; objects with small $W_{\text {dens }}$ and small $A_{\text {iso }}$ as faint and compact objects, usually background galaxies, small stars or spurious detections. Stars describe a locus which is approximately a straight line on the log-log plane (the range between $W_{\text {dens }} \sim 10^{2}$ and $W_{\text {dens }} \sim 10^{5}$ in Fig. 1). Galaxies, saturated sources and other diffuse objects are located to the right of the stellar locus. We define a region where possible LSBG candidates can be found: $W_{\text {dens }}<10^{2}$ (this value corresponding to $\mu_{0} \sim 2.5$ mags brighter than $\sigma_{s k y}$, that is $\approx 22.5 \mathrm{mag} \mathrm{arcsec}^{-2}$ for $\mathrm{g}$ band in SDSS), and beyond $3 \sigma$ from the stellar locus to the right (the two thick dashed lines in Fig. 1 ).
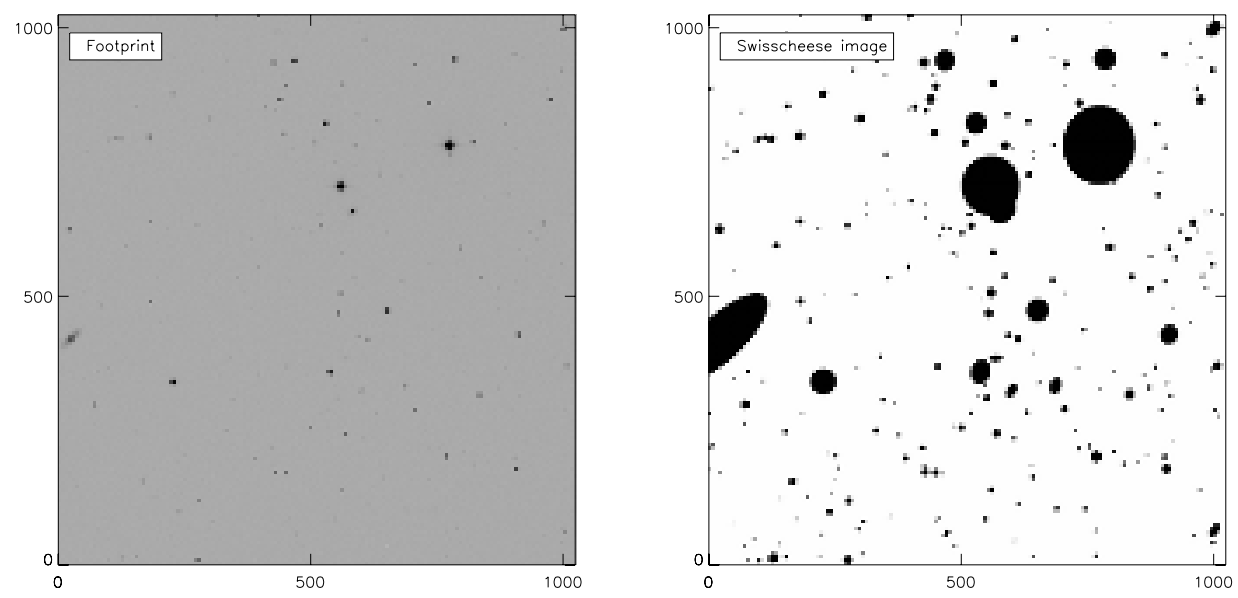

Figure 2: Form a footprint (left) we obtain a "swisscheese" masking map (right) generated using the masked regions of the non interesting objects in Fig. 1.

All the objects that are outside this region are not considered as possible LSBG candidates and are therefore masked on the original image by replacing the area with a noise patch. We keep track of masked regions by generating a "swisscheese" masking map, where pixels belonging to them are set to zero and all the other pixels to unity. This map (see Fig. 2) is then used at a later stage for completeness purposes. The whole procedure is automatically iterated on a footprint-by-footprint basis and is thus homogeneous with respect to different footprints, which have slightly different stellar locii depending on image quality and seeing. 


\subsection{Kernels, candidate significance and parameter estimates}

Convolution by a fixed size kernel yields a map in which coherent positive deviations, even if of low amplitude, are enhanced with respect to the noise in the convolved image: essentially if the flux of the object, $F$, is spread over $N_{o b j}$ independent pixels, then the relative statistical significance in terms of standard deviations with respect to pixel noise gains a factor of $\sqrt{N_{o b j}}$. If we ideally consider Poisson statistics and examine an object covering an area of $N_{o b j}$ pixels over an image with uncorrelated noise per pixel $\sigma_{s k y}$, then the signal to noise in the case of background subtracted faint signals which are much below the sky average level is: $S / N=\sum_{i} S_{i} / \sqrt{\sum_{i} S_{i}+N_{o b j} \sigma_{s k y}^{2}} \simeq \sum_{i} S_{i} / \sqrt{N_{o b j} \sigma_{s k y}^{2}}$ where $S_{i}$ is the signal of pixel $i$ (here $i=1 \ldots N_{o b j}$ ). For a kernel of scale R, the convolved flux is given by $F_{c o n v}(R)=\sum_{j} w_{j} S_{j}$ (with $j=1 \ldots \mathcal{N}$ denotes the pixels covered by the kernel of size $R$ ), where $w_{j}$ are the convolving kernel normalized weights $\left(\sum_{j} w_{j} \equiv 1\right)$. The variance of the convolved field is given by $\Sigma_{F}^{2}(R)=\sigma_{s k y}^{2} \sum_{i}\left(w_{i}^{2}\right) \equiv \mathcal{N}_{\text {eff }} \sigma_{s k y}^{2}$. By dividing the value of $F_{\text {conv }}(R)$ with the standard deviation $\Sigma_{F}$ of the convolved image at the scale $\mathrm{R}$, we obtain the significance of a detection $\nu(R)$, i.e. a convolved $\mathrm{S} / \mathrm{N}$ ratio: $\nu(R) \equiv F_{\text {conv }}(R) / \Sigma_{F}(R)$.

It should be noted that, since the variance is proportional to the area, the denominator typically increases linearly with the scale while the numerator increases approximately as $1-\exp (r / \alpha)$, for objects with exponential profiles; this means that the ratio $\nu(r)$ has a well defined maximum at a radius $r_{\text {Max }}\left(\nu_{\text {Max }}=\nu\left(r_{\text {Max }}\right)\right)$ which is a function of the ratio $\alpha / r$ (Irwin et al. 1990). The maximum significance for a top-hat kernel is reached when the kernel scale $\mathrm{R}$ is approximately twice the scale length $\alpha$, actually $1.8 \alpha$. The result of the convolution process $\left(F_{\text {conv }}\right)$ of a typical LSBG exponential profile with a set of circular top-hat kernels, is a function of kernel dimension $\mathrm{R}$, galaxy scale length $\alpha$ and galaxy total flux. By varying the kernel dimension $\mathrm{R}$, the amount of convolved flux, for a galaxy with scale length $\alpha$, increases with $R$ and becomes a constant when it reaches the maximum value corresponding to the total flux of the galaxy. Therefore from the value of $\nu_{\text {Max }}$ an estimate of scale length and central surface brightness of the candidate LSBG can be obtained.

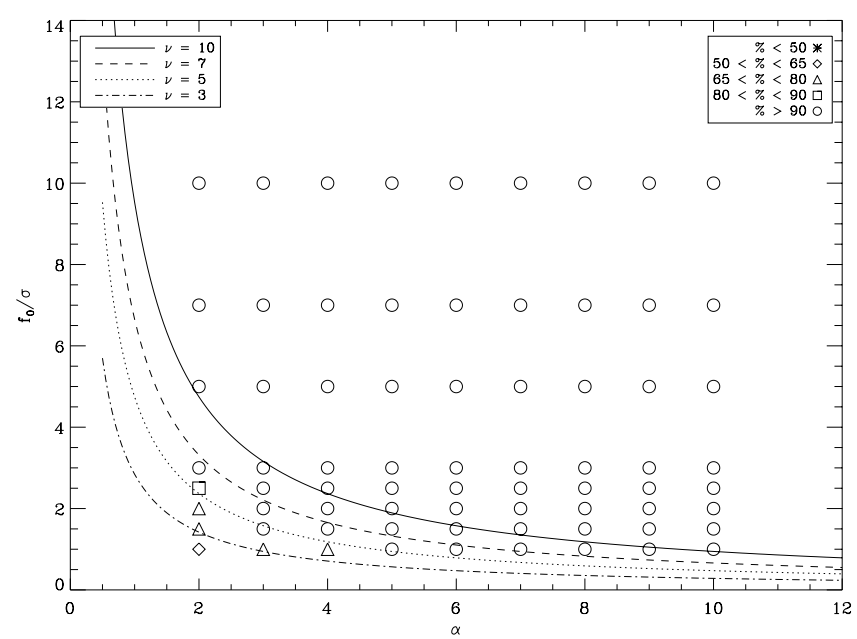

Figure 3: Percentage of recovered objects from simulations (found/total) for different scale lengths $\alpha$ and peak flux $f_{0}$, in number of sigma. Superimposed curves show the trend for different signal-to-noise ratio. 
For each pixel on the original image, the procedure compares the significance of the detection on the stack of kernels and produces a final map where the most significant value (i.e. the one with the greatest value of $\nu(R)$ ) is kept. In a different array, for each pixel, the kernel scale corresponding to the maximum value of $\nu$ is stored. We use the former (i.e. the final single "significance map") for galaxy detection and the latter for the estimation of galaxy structural parameters. The significance for ideal objects is easily derived as $\nu(R) \simeq\left\{2 \pi \alpha^{2} f_{0}\left[1-(1+R / \alpha) e^{-R / \alpha}\right]\right\} /\left(\sqrt{\pi} R \sigma_{s k y}\right)$. Therefore we can identify iso-significance curves for different values of $\nu$ (see Fig. 3$)$ in the $\left(\alpha, f_{0}\right)$ plane. By fixing the size at maximum $\nu$ to be $R_{M a x}=1.8 \alpha$, we can give an estimate of $f_{0}$ for different $\nu$ values.

A previous version of this method was successfully used in the Virgo INT survey (Sabatini et al. (2003)). In the current version we have also implemented direct automatic fits to brightness radial profiles of the significant candidates. This also help in discarding the majority of contaminants, due to positive perturbations which are not due to centrally condensed objects. An example of a large and prominent LSB is shown in Fig. 4.
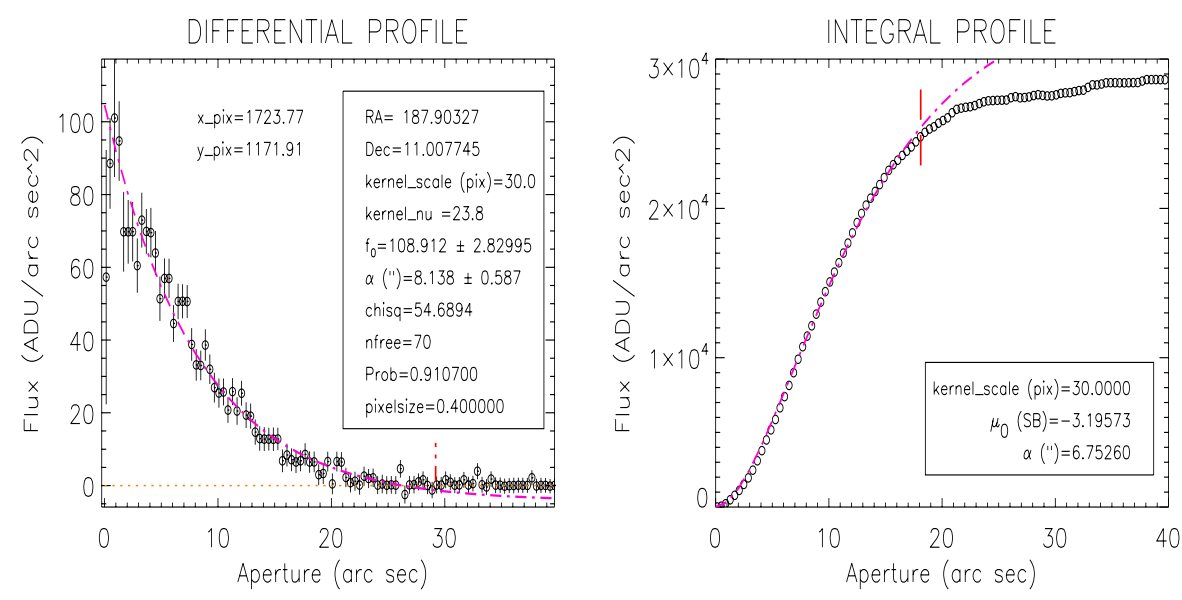

Figure 4: Radial fits to differential and integral flux profiles for a prominent SDSS candidate.

\section{Acknowledgements}

We thank U. Becciani (Catania Observatory) for assistance in porting the code in a GRID environment and the collaborators of the previous analyses of DPOSS plates.

\section{References}

Bertin, E., \& Arnouts, S., 1996, A\&AS, 117, 393

Disney, M., 1976, Nature, 263, 573

Haberzettl, et al. 2007, astro-ph 0701707 (see also poster at this conference)

Kniazev, et al. 2004, ApJ 127, 704

Irwin, M., 1985, MNRAS, 214, 575

Irwin, M., Davies, J. I., Disney, M. J., \& Phillips, S., 1990, MNRAS, 245, 289

Sabatini, S., Davies, J. I., Scaramella, R., Smith, R., Baes, M., Linder, S. M., Roberts, S., \& Testa, V., 2003, MNRAS 341, 981. 